\title{
Bipolar-valued hesitant fuzzy graph and its application
}

\author{
Sakshi Dev Pandey ${ }^{1} \cdot$ A. S. Ranadive ${ }^{1} \cdot$ Sovan Samanta ${ }^{2}$ (I) \\ Received: 20 June 2021 / Revised: 20 August 2021 / Accepted: 28 September 2021 / Published online: 1 December 2021 \\ (c) The Author(s), under exclusive licence to Springer-Verlag GmbH Austria, part of Springer Nature 2021
}

\begin{abstract}
In the real-world scenario, one has to find a dominating person in a social network, conferences, meetings or any group discussion. The fuzzy graph (network) is one of the most powerful tools to find the strongest influential person in a network. This paper aims to develop a concept of fuzzy graphs (FGs) in the setup of bipolar-valued hesitant fuzzy sets (BVHFs). The concept of bipolar-valued hesitant fuzzy graph (BVHFG) is different from the concept of bipolar fuzzy graph (BFG). BVHFG is the generalization of hesitant fuzzy graph (HFG), which not only considers the satisfaction degree of units in a network but also considers the satisfaction degree to some implicit counter property of units with several bipolar fuzzy values. We first introduce the definition of BVHFG, represented by another class of imprecise membership grades that refers to BVHF membership grades. We shall subsequently see the scope of BVHF membership grades in BVHFG is greater than the scope of bipolar-valued membership grades in BFG. In addition, we also discuss the basic operations and functional properties of BVHFGs. Finally, we propose a numerical method to find the most dominating person using our proposed work. As the proposed method of ranking considers the degree of hesitation as well as bipolarity, this method has the edge over earlier work. To establish the importance of our method, we also find domination degrees for HFG and BVHFG using the same example and show that there is a significant change in the ranking of dominating persons.
\end{abstract}

Keywords Bipolar-valued hesitant fuzzy sets · Bipolar-valued hesitant fuzzy graph · Directed bipolar-valued hesitant fuzzy graph $\cdot$ Domination degree

\section{Introduction}

Fuzzy set theory introduced by Klir and Yuan (1996) can be seen as a body of concepts that deal with the kinds of uncertainties which arise when the class boundary is not properly defined. A fuzzy set is basically represented by its membership function which is a generalization of the characteristic function. After the development of fuzzy sets, it has become the most popular research field in various disciplines. Several generalizations of fuzzy sets have been introduced in

Sovan Samanta

ssamantavu@gmail.com

Sakshi Dev Pandey

sdpandey.in@gmail.com

A. S. Ranadive

asranadive04@yahoo.co.in

1 Department of Mathematics, Guru Ghasidas Vishwavidyalya Bilaspur, Chhattisgarh, India

2 Department of Mathematics, Tamralipta Mahavidyalaya, Tamluk, West Bengal 721636, India the field of research, for example, Zhang (1994) presented bipolar-valued fuzzy sets, as an extension of fuzzy sets whose membership lies within $[-1,1]$. In the bipolar-valued fuzzy set, membership degree 0 indicates that the element has no relevance to the given condition, membership degree $(0,1]$ indicates the satisfaction degree of the element corresponding to some given properties, membership degree $[-1,0)$ indicates the degree of satisfaction of an element for some inherent inverse properties of the given condition. In this generalization, we assume that it is dealing with the degree of satisfaction of the elements that satisfy the corresponding property and some inherent counter property of the given condition, but there is no consideration for hesitation on this satisfaction degree. This generalization is still not more-convenient with real-world problems. Therefore, Mandal and Ranadive (2019) propose the concept of bipolarvalued hesitant fuzzy set, and it deals the situation in bipolar membership degree with hesitation index.

Graph theory originated in 1736 for Euler's solution to the Königsberg bridge puzzle, provides a powerful tool for solving problems in various disciplines such as computer 
analysis, data structure, mathematical modeling, economics, engineering, social sciences, and statistics. In many cases, some aspects of the graph-theory problems are uncertain, for example, ranking of attributes, group decision-making problems, consistency of preference relation problems, etc. In order to solve such problems, Rosenfeld gave the concept of fuzzy graph, inspired by Kauffman's (1973) fundamental ideas. Currently, a lot of work is being done in the research field using fuzzy graphs, such as, link prediction in social networks (Mahapatra et al. 2019, 2020), generalized neutrosophic planar graphs (Mahapatra et al. 2021), coloring of covid-19 affected regions (Mahapatra et al. 2021, 2020), radio fuzzy graphs (Mahapatra et al. 2019), and packaged food smart traceability and communication (Mahapatra et al. 2020). The concept of fuzzy homomorphism, fuzzy isomorphism, fuzzy co-weak isomorphism was given by Bhutani (1989). After the concept of fuzzy graphs, this area has been variously generalized. Based on the basic idea of Zhang (1994) bipolar fuzzy set theory, Akram (2011, 2013, 2016), Akram and Dudek (2012) proposed the notion of bipolar fuzzy graphs to deal with the bipolarity of real-world problems and gave some basic operations such as Cartesian product, composition and union of two bipolar fuzzy graphs. The book by Akram, Sarwar and Dudek (2021) entitled Graphs for the Analysis of Bipolar Fuzzy Information is an excellent source for research in fuzzy graphs. The features of bipolar fuzzy graph are very useful in the application of decision-making problems (Akram and Waseem 2018). More information on BFGs, irregular BFGs, and bipolar fuzzy hypergraphs can be found in Samanta and Pal (2012a), (b); Samanta et al. (2014); Rashmanlou et al. (2015); Akram and Akmal (2016); Alghamdi et al. (2018). In fuzzy graph theory, each vertex is characterized by membership degrees only, but this theory is not more convenient for modelling some real-life problems. Therefore, Karaaslan (2019) provided the definition of hesitant fuzzy graph whose basic idea was introduced by Torra (2010).

In this paper, we establish the concept of BVHFG with a different approach from BFG. Here we formulate the many real-world problems in a network with several bipolar-valued hesitant membership grades, more reliable with the decision process and many other problems. In this sequence, we also introduce some basic operations such as Cartesian product, strong product and union of two BVHFGs, we also investigate the concept of homomorphism, isomorphism, weak isomorphism, co-weak isomorphism of these graphs. In the final section, we illustrate by mean of an example, finding the most dominating person in the meeting and compare the usage of BVHFG and HFG.

The content of the paper is organized as follows. Section two reviews some basic knowledge on graphs, fuzzy graphs and BVHFSs. Section three refers to the definition and example of BVHFGs, then some common operational laws along with mapping relationships of BVHFGs and propositions are explored. Section four develops a two-stage algorithmic rule by virtue of BVHFGs, and then, a numerical instance concerning finding the most influential person is given. In section five, we tend to study the comparative analysis between BVHFG and HFG to demonstrate the relevancy and effectiveness of the conferred notion of BVHFG. At last, Section six concludes the total paper and points out the significance of BVHFG.

\section{Preliminaries}

In this segment, we cover some basic definitions based on graphs, digraphs, fuzzy graphs and bipolar-valued hesitant fuzzy sets (BVHFSs).

\subsection{Basic definitions on graphs and fuzzy graphs}

Definition 1 (Bollobás 2013) A graph is an ordered pair $G=(V, E)$, where $V$ is the set of vertices and $E$ is the set of edges of $G$ formed by a pair of vertices $E \subset\{(x, y) \mid x, y \in V\}$.

Let $G_{1}=\left(V_{1}, E_{1}\right)$ and $G_{2}=\left(V_{2}, E_{2}\right)$ be two graphs, then the following operations are defined as:

- Cartesian product of two graphs $G_{1}$ and $G_{2}$ denoted by $G_{1} \times G_{2}=\left\{V_{1} \times V_{2}, E_{1} \times E_{2}\right\}$ is defined as $V_{1} \times V_{2}=\left\{\left(v_{1}, v_{2}\right) \mid v_{1} \in V_{1}\right.$ and $\left.v_{2} \in V_{2}\right\}$ and $E_{1} \times E_{2}$ $=\left\{\left(a, a_{2}\right)\left(a, b_{2}\right) \mid a \in V_{1}, a_{2} b_{2} \in E_{2}\right\} \cup\left\{\left(a_{1}, c\right)\left(b_{1}, c\right) \mid a_{1} b_{1}\right.$ $\left.\in E_{1}, c \in V_{2}\right\}$.

- Strong product of two graphs $G_{1}$ and $G_{2}$ denoted by $G_{1} \otimes G_{2}=\left(V_{1} \otimes V_{2}, E_{1} \otimes E_{2}\right)$ is defined as $V_{1} \times V_{2}=\left\{\left(v_{1}, v_{2}\right) \mid v_{1} \in V_{1}, v_{2} \in V_{2}\right\}$ and $E_{1} \times E_{2}=$ $\left\{\left(a, a_{2}\right)\left(a, b_{2}\right) \mid a \in V_{1}, a_{2} b_{2} \in E_{2}\right\} \cup\left\{\left(a_{1}, c\right)\left(b_{1}, c\right) \mid a_{1} b_{1}\right.$ $\left.\in E_{1}, c \in V_{2}\right\} \cup\left\{\left(a_{1}, a_{2}\right)\left(b_{1}, b_{2}\right) \mid a_{1} b_{1} \in E_{1}, a_{2} b_{2} \in E_{2}\right\}$.

Definition 2 A graph $G=(V, E)$ whose edges have the orientations and arrows on edges are used to represent the directions is called directed graph (digraph), denoted by $\overrightarrow{\mathbf{G}}_{d}=(V, \overrightarrow{\mathbf{E}})$.

Definition 3 (Rosenfeld 1975) A fuzzy graph $G^{*}=(V, \mu, \nu)$ over the graph $G=(V, E)$ is characterized by membership functions $\mu: V \rightarrow[0,1]$ and $v: V \times V \rightarrow[0,1]$ such that

$v\left(v_{1} v_{2}\right) \leq \mu\left(v_{1}\right) \wedge \mu\left(v_{2}\right) \forall v_{1}, v_{2} \in V$,

where $\mu\left(v_{1}\right)$ and $v\left(v_{1} v_{2}\right)$ represent the membership value of the vertex $v_{1}$ and edge $v_{1} v_{2}$ in $G^{*}$, respectively. 
Definition 4 (Mordeson and Nair 2012) Let $G^{*}=(V, \mu, v)$ be a fuzzy graph, then complimentary fuzzy graph $\bar{G}^{*}$ of a fuzzy graph $G^{*}$ has the same vertices as $G^{*}$, two vertices are adjacent in $\bar{G}^{*}$ if and only if they are not adjacent in $G^{*}$.

Definition 5 (Poulik and Ghorai 2020) Let $G^{*}=(V, \mu, \nu)$ be a fuzzy graph, then degree of vertex $v_{1}$ in a fuzzy graph $G^{*}$ is defined as $\operatorname{deg}\left(v_{1}\right)=\sum_{v_{1} \neq v \in V} v\left(v_{1} v\right)$.

\subsection{Bipolar-valued hesitant fuzzy set}

Definition 6 (Mandal and Ranadive 2019). Let $X$ be frame of reference. A bipolar-valued hesitant fuzzy set (BVHFS) $A$ on $X$ is defined as:

$A=\{<x, A(x)>\mid x \in X\}$,

where $A(x)$ is a set of some values in $[0,1] \times[-1,0]$. For convenience, we call $A(x)$ a bipolar-valued hesitant fuzzy element (BVHFE) expressed as:

$A(x)=\left\{a_{x} \mid a_{x} \in[0,1] \times[-1,0]\right\}$,

here $a_{x}=\left(a_{x}^{P}, a_{x}^{N}\right)$ is a bipolar-valued fuzzy number (BVFN) such that $a_{x}^{P} \in[0,1]$ and $a_{x}^{N} \in[-1,0]$.

Definition 7 (Mandal and Ranadive 2019) Let X be universe of discourse and for $x \in X$ let $A(x), A_{1}(x), A_{2}(x)$ be the BVHFE's, then

- $A_{1}(x) \cup A_{2}(x)=\left\{\left(\max \left(a_{1 x}^{P}, a_{2 x}^{P}\right), \min \left(a_{1 x}^{N}, a_{2 x}^{N}\right)\right) \mid\right.$

$\left.\mid a_{1 x} \in A_{1}(x), a_{2 x} \in A_{2}(x)\right\}$;

- $A_{1}(x) \cap A_{2}(x)=\left\{\left(\min \left(a_{1 x}^{P}, a_{2 x}^{P}\right), \max \left(a_{1 x}^{N}, a_{2 x}^{N}\right)\right)\right.$

$\left.\mid a_{1 x} \in A_{1}(x), a_{2 x} \in A_{2}(x)\right\}$

- $A(x)^{c}=\left\{\left(1-a_{x}^{P},-1-a_{x}^{N}\right) \mid a_{x} \in A(x)\right\}$.

Definition 8 (Mandal and Ranadive 2019) Let $a_{x}=\left(a_{x}^{P}, a_{x}^{N}\right) \in A(x)$ be a BVFN, and the value of score $s\left(a_{x}\right)$ is defined as:

$s\left(a_{x}\right)=\frac{1}{2}\left(a_{x}^{P}-a_{x}^{N}\right)$,

which is the mean of the satisfaction degree corresponding to some given property of attribute and satisfaction degree to some implicit counter property of attribute of an element.

Definition 9 (Mandal and Ranadive 2019) Let $A(x)$ be a BVHFE, and the score function $s(A(x))$ is defined as:

$s(A(x))=\frac{1}{l(A(x))} \sum_{a_{x} \in A(x)} s\left(a_{x}\right)$,

here $l(A(x))$ denote the number of bipolar values in $A(x)$, and $a_{x}$ is the element in $A(x)$, taken as the form of BVFN.
Inspired by the basic idea of Deepak and John (2014), we define score-based intersection and union of two BVHFSs as follows:

Definition 10 Let A and B be two BVHFSs over X. Then, score-based intersection and union of two BVHFE's $A(x)$ and $B(x)$ are represented by $A(x) \tilde{\wedge} B(x)$ and $A(x) \tilde{V} B(x)$, respectively, which is characterized by

$A(x) \tilde{\wedge} B(x)= \begin{cases}A(x), & \text { if } s(A(x))<s(B(x)), \\ B(x), & \text { if } s(B(x))<s(A(x)), \\ A(x) \text { or } B(x), & \text { if } s(A(x))=s(B(x)),\end{cases}$

and

$A(x) \tilde{V} B(x)= \begin{cases}A(x), & \text { if } s(A(x))>s(B(x)), \\ B(x), & \text { if } s(B(x))>s(A(x), \\ A(x) \text { or } B(x), & \text { if } s(A(x))=s(B(x)) .\end{cases}$

The following is a trivial consequence of our definition.

Proposition 1 Let $A$ and $B$ be two BVHFSs over the nonempty set $X$. Then, $s(A(x) \widetilde{\wedge} B(x))=s(A(x)) \wedge s(B(x))$ and $s(A(x) \tilde{\vee} B(x))=s(A(x)) \vee s(B(x))$.

\subsection{Bipolar-valued hesitant fuzzy relation}

Definition 11 Let $A$ and $B$ be two BVHFSs over the nonempty set $X$. Then, the score-based Cartesian product of two BVHFSs $\mathrm{A}$ and $\mathrm{B}$ is represented by $A \tilde{\times} B$ and characterized by

$$
\begin{aligned}
A \tilde{\times} B & =\{<(x, y),(A \tilde{\times} B)(x, y)>\mid(x, y) \in X \times X\}, \\
& =\{<(x, y), A(x) \tilde{\times} B(y)>\mid(x, y) \in X \times X\} .
\end{aligned}
$$

Definition 12 Let $\mathrm{X}$ be a non-empty set. Let $A$ and $B$ be two BVHFSs on $\mathrm{X}$, for $x, y \in X$, let $A(x, y): X \times X \rightarrow P([0,1] \times[-1,0])$ be bipolar-valued hesitant fuzzy relation on $X$, and then, we call $A$ is score-based bipolar-valued hesitant fuzzy relation on $B$ if $s(A(x, y)) \leq s(B(x)) \wedge s(B(y))$ for all $x, y \in X$.

\section{Bipolar-valued hesitant fuzzy graph}

In this section, we define the notion of bipolar-valued hesitant fuzzy graph (BVHFG) using the score function, which is the mean of satisfaction degree of an element corresponding to some given attribute property and some implicit counter property of attribute. In addition, we give some examples, basic operations and define the isomorphisms on BVHFGs. 
Definition 13 Let $G=(V, E)$ be a graph. A bipolarvalued hesitant fuzzy graph (BVHFG) with $V$ as a reference set is a pair $G^{*}=(A, B)$ where $A$ and $B$ are BVHFSs in $V$ and $\tilde{V}^{2}$ respectively, which is characterized by membership functions $A: V \rightarrow P([0,1] \times[-1,0])$ and $B: \tilde{V}^{2} \rightarrow P([0,1] \times[-1,0])$ with the condition

$$
\begin{gathered}
s(B(x y)) \leq s(A(x)) \wedge s(A(y)) \forall x y \in \tilde{V}^{2} \\
\text { and } s(B(x y))=0 \forall x y \in\left(\tilde{V}^{2}-E\right),
\end{gathered}
$$

here $B(x y)$ and $A(x)$ are BVHFEs defined as $B(x y)=\left\{\left(b_{x y}^{P}, b_{x y}^{N}\right) \mid\left(b_{x y}^{P}, b_{x y}^{N}\right) \in[0,1] \times[-1,0]\right\} \quad$ a n d $A(x)=\left\{\left(a_{x}^{P}, a_{x}^{N}\right) \mid\left(a_{x}^{P}, a_{x}^{N}\right) \in[0,1] \times[-1,0]\right\}$.

\section{Remark 1}

- If we neglect the negative membership degrees of vertices in a network, which represents the satisfaction degrees to some implicit counter property of attribute, then BVHFG reduces to HFG (Karaaslan 2019).

- The concept of bipolar-valued hesitant fuzzy graph differs from the concept of bipolar fuzzy graph in its approach to the definition of 13 .

- From definition 13 , we can easily understand that the class of imprecise membership grades in BVHFG is greater than the class of imprecise membership grades in BFG. Membership grades of bipolar fuzzy graphs also satisfy the condition of BVHFG, but the converse is not always true. Here in Fig. 1 all membership grades satisfy the condition of BVHFG as in definition 13. However, for the edge between vertex $A$ and vertex $B$, the positive satisfaction degree $0.5 \not \leq \min (0.4,0.8)$ and negative satisfaction degree $-0.4 \nsupseteq \max (-0.6,-0.3)$, i.e., it does not satisfy the condition of BFG. Hence, the class of membership grades of BVHFG is larger than the class of membership grades in BFG.

Example 1 Suppose there are six participants in the debate competition program and board members select the one participant as a winner according to the four functional properties, that is, influence power, conversation style, knowledge

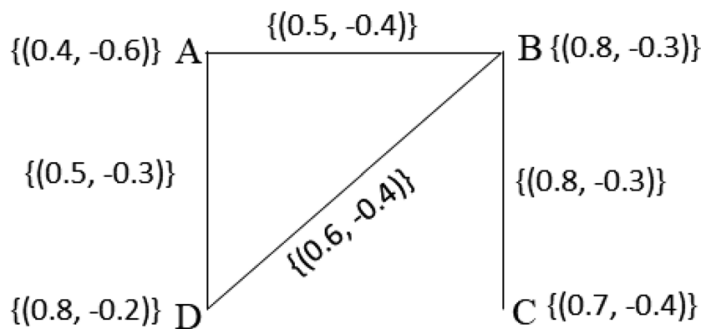

Fig. 1 BVHFG Vs BFG of the given topic, speech ingenuity. Instead of providing a traditional decision matrix, board members comprehensively evaluate the correlation of four properties between six participants. Let $V$ be a set of six participants $\left\{v_{1}, v_{2}, v_{3}, v_{4}, v_{5}, v_{6}\right\}$ and $E=\left\{v_{1} v_{2}, v_{1} v_{4}, v_{2} v_{3}, v_{2} v_{5}, v_{3} v_{4}, v_{4} v_{5}, v_{5} v_{6}, v_{6} v_{1}\right\}$ be the correlation of four properties among participants, then BVHFG and BVHFSs $\mathrm{A}$ and $\mathrm{B}$ over $\mathrm{V}$ and $\tilde{V}^{2}$ are given in Fig. 2 and Table 1, respectively:

Definition 14 Let $G^{*}=(A, B)$ be a BVHFG over the graph $G=(V, E)$, score-based degree of a vertex $v_{1} \in V$ in BVHFG is denoted by $\mathfrak{D}\left(v_{1}\right)$ and defined as $\mathfrak{D}\left(v_{1}\right)=\sum_{v \neq v_{1} \in V} s\left(B\left(v_{1} v\right)\right)$.

Example 2 For Example 1, we calculate the score-based degree of each vertex in BVHFG and find that $\mathfrak{D}\left(v_{1}\right)=1.200$, $\mathfrak{D}\left(v_{2}\right)=1.233, \mathfrak{D}\left(v_{3}\right)=0.425, \mathfrak{D}\left(v_{4}\right)=1.275, \mathfrak{D}\left(v_{5}\right)=1.35$, $\mathfrak{D}\left(v_{6}\right)=0.892$.

Definition 15 Let $G_{1}^{*}=\left(A_{1}, B_{1}\right)$ and $G_{2}^{*}=\left(A_{2}, B_{2}\right)$ be two BVHFGs over the graph $G=(V, E)$ then we say that $G_{1}^{*}$ is score-based BVHF-subgraph of $G_{2}^{*}$ if it satisfies the conditions

$s\left(A_{1}(x)\right) \leq s\left(A_{2}(x)\right), s\left(B_{1}(x y)\right) \leq s\left(B_{2}(x y)\right)$

$\forall x \in V, \forall x y \in \tilde{V}^{2}$.

\subsection{Basic operations on BVHFGs}

Let $G_{1}^{*}=\left(A_{1}, B_{1}\right)$ and $G_{2}^{*}=\left(A_{2}, B_{2}\right)$ be two BVHFGs over the graphs $G_{1}=\left(V_{1}, E_{1}\right)$ and $G_{2}=\left(V_{2}, E_{2}\right)$ respectively. We now give some operations and related results for BVHFGs:

1. Cartesian product: Cartesian product of two BVHFGs denoted by $G_{1}^{*} \widetilde{\times} G_{2}^{*}=\left(A_{1} \widetilde{\times} A_{2}, B_{1} \widetilde{\times} B_{2}\right)$ is defined as:

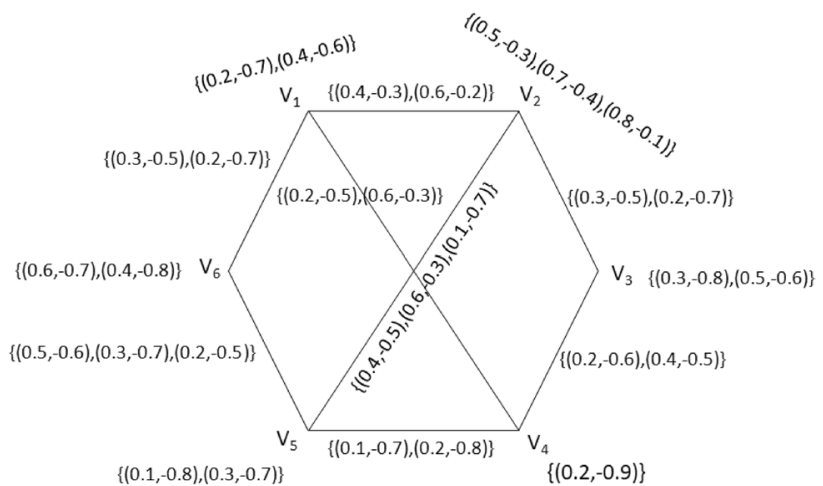

Fig. 2 Bipolar-valued hesitant fuzzy graph (BVHFG) 
Table 1 Bipolar-valued hesitant fuzzy table

\begin{tabular}{lllll}
\hline & $V_{1}$ & $V_{2}$ & $V_{3}$ & $V_{4}$ \\
\hline A & $\{(0.2,-0.7),(0.4,-0.6)\}$ & $\{(0.5,-0.3),(0.7,-0.4),(0.8,-0.1)\}$ & $\{(0.3,-0.8),(0.5,-0.6)\}$ & $\{(0.2,-0.9)\}$ \\
Score & 0.475 & 0.467 & 0.55 & 0.55 \\
\hline & $V_{5}$ & $V_{6}$ & \\
\hline & $\{(0.1,-0.8),(0.3,-0.7)\}$ & $\{(0.6,-0.7),(0.4,-0.8)\}$ & $V_{2} V_{5}$ \\
\hline & 0.475 & 0.625 & $\{(0.4,-0.5),(0.6,-0.3),(0.1,-0.7)\}$ \\
\hline B & $V_{1} V_{2}$ & $V_{1} V_{4}$ & $V_{2} V_{3}$ & 0.433 \\
\hline & $\{(0.4,-0.3),(0.6,-0.2)\}$ & $\{(0.2,-0.5),(0.6,-0.3)\}$ & $\{(0.3,-0.5),(0.2,-0.7)\}$ & $V_{6} V_{1}$ \\
\hline & 0.375 & 0.400 & 0.425 & $\{(0.3,-0.5),(0.2,-0.7)\}$ \\
\hline
\end{tabular}

- $\left(A_{1} \widetilde{\times} A_{2}\right)\left(x_{1}, x_{2}\right)=A_{1}\left(x_{1}\right) \tilde{\wedge} A_{2}\left(x_{2}\right) \forall\left(x_{1}, x_{2}\right) \in V_{1} \times V_{2}$.

- $\left(B_{1} \widetilde{\times} B_{2}\right)\left(\left(x, x_{2}\right)\left(x, y_{2}\right)\right)=A_{1}(x) \widetilde{\wedge} B_{2}\left(x_{2} y_{2}\right) \forall x \in V_{1}, x_{2} y_{2} \in E_{2}$.

- $\left(B_{1} \widetilde{x} B_{2}\right)\left(\left(x_{1}, z\right)\left(y_{1}, z\right)\right)=B_{1}\left(x_{1} y_{1}\right) \widetilde{\wedge} A_{2}(z) \forall x_{1} y_{1} \in E_{1}, z \in V_{2}$.

Proposition 2 Let $G_{1}^{*}$ and $G_{2}^{*}$ be two BVHFGs, then $G_{1}^{*} \widetilde{\times} G_{2}^{*}$ is a $B V H F G$.

Proof For any $x \in V_{1}$ and $x_{2} y_{2} \in E_{2}$ we have,

$$
\begin{aligned}
& s\left(\left(B_{1} \widetilde{\times} B_{2}\right)\left(\left(x, x_{2}\right)\left(x, y_{2}\right)\right)\right) \\
& \quad=s\left(A_{1}(x) \widetilde{\wedge} B_{2}\left(x_{2} y_{2}\right)\right) \\
& \quad=s\left(A_{1}(x)\right) \wedge s\left(B_{2}\left(x_{2} y_{2}\right)\right) \\
& \quad \leq s\left(A_{1}(x)\right) \wedge\left(s\left(A_{2}\left(x_{2}\right)\right) \wedge s\left(A_{2}\left(y_{2}\right)\right)\right) \\
& \quad=\left(s\left(A_{1}(x)\right) \wedge s\left(A_{2}\left(x_{2}\right)\right)\right) \wedge\left(s\left(A_{1}(x) \wedge s\left(A_{2}\left(y_{2}\right)\right)\right)\right. \\
& \quad=s\left(A_{1}(x) \tilde{\wedge} A_{2}\left(x_{2}\right)\right) \wedge s\left(A_{1}(x) \widetilde{\wedge} A_{2}\left(y_{2}\right)\right) \\
& \quad=s\left(\left(A_{1} \widetilde{\times} A_{2}\right)\left(x, x_{2}\right)\right) \wedge s\left(\left(A_{1} \widetilde{\times} A_{2}\right)\left(x, y_{2}\right)\right) .
\end{aligned}
$$

For any $x_{1} y_{1} \in E_{1}$ and $z \in V_{2}$ we have,

$$
\begin{aligned}
s( & \left.\left(B_{1} \widetilde{\times} B_{2}\right)\left(\left(x_{1}, z\right)\left(y_{1}, z\right)\right)\right) \\
& =s\left(B_{1}\left(x_{1} y_{1}\right) \widetilde{\wedge} A_{2}(z)\right) \\
& =s\left(B_{1}\left(x_{1} y_{1}\right)\right) \wedge s\left(A_{2}(z)\right) \\
& \leq\left(s\left(A_{1}\left(x_{1}\right)\right) \wedge s\left(A_{1}\left(y_{1}\right)\right)\right) \wedge s\left(A_{2}(z)\right) \\
& =\left(s\left(A_{1}\left(x_{1}\right)\right) \wedge s\left(A_{2}(z)\right)\right) \wedge\left(s\left(A_{1}\left(y_{1}\right) \wedge s\left(A_{2}(z)\right)\right)\right. \\
& =s\left(A_{1}\left(x_{1}\right) \tilde{\wedge} A_{2}(z)\right) \wedge s\left(A_{1}\left(y_{1}\right) \tilde{\wedge} A_{2}(z)\right) \\
& =s\left(A_{1} \widetilde{\times} A_{2}\right)\left(x_{1}, z\right) \wedge s\left(\left(A_{1} \widetilde{\times} A_{2}\right)\left(y_{1}, z\right)\right) .
\end{aligned}
$$

2. Strong product: Strong product of two BVHFGs denoted by $G_{1}^{*} \widetilde{\otimes} G_{2}^{*}=\left(A_{1} \widetilde{\otimes} A_{2}, B_{1} \widetilde{\otimes} B_{2}\right)$ is defined as:
- $\left(A_{1} \widetilde{\otimes} A_{2}\right)\left(x_{1}, x_{2}\right)=A_{1}\left(x_{1}\right) \widetilde{\wedge} A_{2}\left(x_{2}\right) \forall\left(x_{1}, x_{2}\right) \in V_{1} \times V_{2}$.

- $\left(B_{1} \widetilde{\otimes} B_{2}\right)\left(\left(x, x_{2}\right)\left(x, y_{2}\right)\right)=A_{1}(x) \widetilde{\wedge} B_{2}\left(x_{2} y_{2}\right) \forall x \in V_{1}, x_{2} y_{2} \in E_{2}$.

- $\left(B_{1} \widetilde{\otimes} B_{2}\right)\left(\left(x_{1}, z\right)\left(y_{1}, z\right)\right)=B_{1}\left(x_{1} y_{1}\right) \widetilde{\wedge} A_{2}(z) \forall x_{1} y_{1} \in E_{1}, z \in V_{2}$.

- $\left(B_{1} \widetilde{\otimes} B_{2}\right)\left(\left(x_{1}, x_{2}\right)\left(y_{1}, y_{2}\right)\right)=B_{1}\left(x_{1} y_{1}\right) \widetilde{\wedge} B_{2}\left(x_{2} y_{2}\right) \forall x_{1} y_{1} \in E_{1}, x_{2} y_{2} \in E_{2}$.

Proposition 3 Let $G_{1}^{*}$ and $G_{2}^{*}$ be two BVHFGs, then $G_{1} \widetilde{\otimes} G_{2}$ is a $B V H F G$.

Proof The proof of this proposition is similar as that of proposition 2 and hence, is omitted.

3. Union: Union of two BVHFGs denoted by $G_{1}^{*} \cup G_{2}^{*}=\left(A_{1} \cup A_{2}, B_{1} \cup B_{2}\right)$ s.t. $s\left(A_{1}(x)\right)=0$ if $x \notin V_{1}$ and $s\left(A_{2}(x)\right)=0$ if $x \notin V_{2}$ is defined as:

- $\left(A_{1} \cup A_{2}\right)(x)=A_{1}(x) \widetilde{V} A_{2}(x) \forall x \in V_{1} \cup V_{2}$.

- $\left(B_{1} \cup B_{2}\right)(x y)=B_{1}(x y) \widetilde{V} B_{2}(x y) \forall x y \in E_{1} \cup E_{2}$.

Proposition 4 Let $G_{1}^{*}$ and $G_{2}^{*}$ be two BVHFGs, then $G_{1}^{*} \cup G_{2}^{*}$ is a $B V H F G$.

Proof For any xy $\in E_{1} \cup E_{2}$ we have

$$
\begin{aligned}
s( & \left.\left(B_{1} \cup B_{2}\right)(x y)\right) \\
& =s\left(B_{1}(x y) \widetilde{\vee} B_{2}(x y)\right) \\
& =s\left(B_{1}(x y)\right) \vee s\left(B_{2}(x y)\right) \\
& \leq\left(s\left(A_{1}(x)\right) \wedge s\left(A_{1}(y)\right)\right) \vee\left(s\left(A_{2}(x)\right) \wedge s\left(A_{2}(y)\right)\right) \\
& =\left(s\left(A_{1}(x)\right) \vee s\left(A_{2}(x)\right)\right) \wedge\left(s\left(A_{1}(y)\right) \vee s\left(A_{2}(y)\right)\right) \\
& =s\left(A_{1}(x) \widetilde{\vee} A_{2}(x)\right) \wedge s\left(A_{1}(y) \widetilde{\vee} A_{2}(y)\right) \\
& =S\left(\left(A_{1} \cup A_{2}\right)(x)\right) \wedge s\left(\left(A_{1} \cup A_{2}\right)(y)\right) .
\end{aligned}
$$




\subsection{Isomorphism between bipolar-valued hesitant fuzzy graphs}

Definition 16 Let $G_{1}^{*}$ and $G_{2}^{*}$ be the BVHFGs. A homomorphism $f: G_{1}^{*} \rightarrow G_{2}^{*}$ is a mapping $f: V_{1} \rightarrow V_{2}$ satisfying the following conditions:

- $s\left(A_{1}\left(x_{1}\right)\right) \leq s\left(A_{2}\left(f\left(x_{1}\right)\right)\right) \forall x_{1} \in V_{1}$.

- $s\left(B_{1}\left(x_{1} y_{1}\right)\right) \leq s\left(B_{2}\left(f\left(x_{1}\right) f\left(y_{1}\right)\right)\right) \forall x_{1} y_{1} \in \widetilde{V_{1}^{2}}$.

Definition 17 Let $G_{1}^{*}$ and $G_{2}^{*}$ be the BVHFGs. An isomorphism $f: G_{1}^{*} \rightarrow G_{2}^{*}$ is a bijective mapping $f: V_{1} \rightarrow V_{2}$ satisfying the following conditions:

- $s\left(A_{1}\left(x_{1}\right)\right)=s\left(A_{2}\left(f\left(x_{1}\right)\right)\right) \forall x_{1} \in V_{1}$.

- $s\left(B_{1}\left(x_{1} y_{1}\right)\right)=s\left(B_{2}\left(f\left(x_{1}\right) f\left(y_{1}\right)\right)\right) \forall x_{1} y_{1} \in \widetilde{V_{1}^{2}}$.

Definition 18 Let $G_{1}^{*}$ and $G_{2}^{*}$ be the BVHFGs. Then, a weak isomorphism $f: G_{1}^{*} \rightarrow G_{2}^{*}$ is a bijective mapping $f: V_{1} \rightarrow V_{2}$ satisfying the following conditions:

- $s\left(A_{1}\left(x_{1}\right)\right)=s\left(A_{2}\left(f\left(x_{1}\right)\right)\right) \forall x_{1} \in V_{1}$.

- $s\left(B_{1}\left(x_{1} y_{1}\right)\right) \leq s\left(B_{2}\left(f\left(x_{1}\right) f\left(y_{1}\right)\right)\right) \forall x_{1} y_{1} \in \widetilde{V_{1}^{2}}$.

Definition 19 Let $G_{1}^{*}$ and $G_{2}^{*}$ be the BVHFGs. A coweak isomorphism $f: G_{1}^{*} \rightarrow G_{2}^{*}$ is a bijective mapping $f: V_{1} \rightarrow V_{2}$ satisfying the following conditions:

- $s\left(A_{1}\left(x_{1}\right)\right) \leq\left(A_{2}\left(f\left(x_{1}\right)\right)\right) \forall x_{1} \in V_{1}$.

- $s\left(B_{1}\left(x_{1} y_{1}\right)\right)=s\left(B_{2}\left(f\left(x_{1}\right) f\left(y_{1}\right)\right)\right) \forall x_{1} y_{1} \in \widetilde{V_{1}^{2}}$.

\section{Remark 2}

- If $G_{1}^{*}=G_{2}^{*}=G^{*}$, then the homomorphism f over itself is called an endomorphism. An isomorphism $\mathrm{f}$ over $\mathrm{G}$ is called an automorphism.

- Let $G^{*}$ be a BVHFG of a graph G. Let $\operatorname{Aut}\left(G^{*}\right)$ be the set of all bipolar-valued hesitant automorphism of $G^{*}$. Let $e: G^{*} \rightarrow G^{*}$ be a map defined by $e(x)=x \forall x \in V$. Clearly $e \in \operatorname{Aut}(G)$.
Proposition 5 If $G_{1}^{*}, G_{2}^{*}, G_{3}^{*}$ are BVHFGs, then the isomorphism between these graphs is an equivalence relation.

Proof For reflexivity we can use identity mapping between BVHFGs, and it is trivial. For symmetry, we assume a function $h: V_{1} \rightarrow V_{2}$ is an isomorphism on $G_{1}^{*}$ onto $G_{2}^{*}$ such that $h\left(m_{1}\right)=m_{2} \forall m_{1} \in V_{1}$ with conditions

$$
\begin{aligned}
& s\left(A_{1}\left(m_{1}\right)\right)=s\left(A_{2}\left(h\left(m_{1}\right)\right)\right), s\left(B_{1}\left(m_{1} n_{1}\right)\right) \\
& \quad=s\left(B_{2}\left(h\left(m_{1}\right) h\left(n_{1}\right)\right)\right) \forall m_{1} \in V_{1}, \forall m_{1} n_{1} \in \tilde{V}_{1}^{2} ;
\end{aligned}
$$

further, since $h$ is isomorphism, we have $h^{-1}\left(m_{2}\right)=m_{1} \forall m_{2} \in V_{2}$, satisfies condition (1), we have

$$
\begin{aligned}
& s\left(A_{1}\left(h^{-1}\left(m_{2}\right)\right)\right)=s\left(A_{2}\left(m_{2}\right)\right), s\left(B_{1}\left(h^{-1}\left(m_{2}\right) h^{-1}\left(n_{2}\right)\right)\right) \\
& \left.=s\left(B_{2}\left(m_{2} n_{2}\right)\right)\right) \forall m_{2} \in V_{2}, \forall m_{2} n_{2} \in \tilde{V}_{2}^{2} .
\end{aligned}
$$

Thus, a mapping $h^{-1}: V_{2} \rightarrow V_{1}$ is an isomorphism from $G_{2}$ onto $G_{1}$. For transitivity, we assume $h_{1}: V_{1} \rightarrow V_{2}$ such that $h_{1}\left(m_{1}\right)=m_{2} \forall m_{1} \in V_{1}$ and $h_{2}: V_{2} \rightarrow V_{3}$ such that $h_{2}\left(m_{2}\right)=m_{3} \forall m_{2} \in V_{2}$ are isomorphisms between $G_{1}$ onto $G_{2}$ and $G_{2}$ onto $G_{3}$, respectively. Further $h_{2} o h_{1}: V_{1} \rightarrow V_{3}$ is a composition of $h_{1}$ and $h_{2}$ such that $\left(h_{2} o h_{1}\right)\left(m_{1}\right)=h_{2}\left(h_{1}\left(m_{1}\right)\right) \forall m_{1} \in V_{1}$.

Since the map $h_{1}: V_{1} \rightarrow V_{2}$ is an isomorphism, we have

$s\left(A_{1}\left(m_{1}\right)\right)=s\left(A_{2}\left(h_{1}\left(m_{1}\right)\right)\right)=s\left(A_{2}\left(m_{2}\right)\right) \forall m_{1} \in V_{1} ;$

$$
s\left(B_{1}\left(m_{1} n_{1}\right)\right)=s\left(B_{2}\left(h_{1}\left(m_{1}\right) h\left(n_{1}\right)\right)\right)
$$$$
=s\left(B_{2}\left(m_{2} n_{2}\right)\right) \forall m_{1} n_{1} \in \tilde{V}_{1}^{2} \text {. }
$$

Again since the map $h_{2}: V_{2} \rightarrow V_{3}$ is an isomorphism, we have

$s\left(A_{2}\left(m_{2}\right)\right)=s\left(A_{3}\left(h_{2}\left(m_{2}\right)\right)\right)=s\left(A_{3}\left(m_{3}\right)\right) \forall m_{2} \in V_{2} ;$

$s\left(B_{2}\left(m_{2} n_{2}\right)\right)=s\left(B_{3}\left(h_{2}\left(m_{2}\right) h\left(n_{2}\right)\right)\right)$

$=s\left(B_{3}\left(m_{3} n_{3}\right)\right) \forall m_{2} n_{2} \in \tilde{V}_{2}^{2}$.

From expressions (2) and (4), we have

$$
\begin{aligned}
s\left(A_{1}\left(m_{1}\right)\right)=s\left(A_{2}\left(h_{1}\left(m_{1}\right)\right)\right)=s\left(A_{2}\left(m_{2}\right)\right)=s\left(A_{3} h_{2}\left(m_{2}\right)\right) & =s\left(A_{3}\left(h_{2}\left(h_{1}\left(m_{1}\right)\right)\right)\right) ; \\
& =s\left(A_{3}\left(h_{2} o h_{1}\left(m_{1}\right)\right)\right) \forall m_{1} \in V_{1} .
\end{aligned}
$$

- If $G_{1}^{*}=G_{2}^{*}$, then the weak and co-weak isomorphism actually become isomorphic.

- If $f: V_{1} \rightarrow V_{2}$ is a bijective map, then $f^{-1}: V_{2} \rightarrow V_{1}$ is also a bijective map. 


$$
\begin{aligned}
& s\left(B_{1}\left(m_{1} n_{1}\right)\right)=s\left(B_{2}\left(h_{1}\left(m_{1}\right) h_{1}\left(n_{1}\right)\right)\right) \\
& \quad=s\left(B_{2}\left(m_{2} n_{2}\right)\right)=s\left(B_{3}\left(h_{2}\left(m_{2}\right) h_{2}\left(n_{2}\right)\right)\right) ; \\
& \quad=s\left(B_{3}\left(h_{2}\left(h_{1}\left(m_{1}\right)\right)\right)\left(h_{2}\left(h_{1}\left(n_{1}\right)\right)\right)\right) ; \\
& \quad=s\left(B_{3}\left(\left(h_{2} o h_{1}\right)\left(m_{1}\right)\left(h_{2} o h_{1}\right)\left(n_{1}\right)\right)\right) \forall m_{1} n_{1} \in \tilde{V}_{1}^{2} .
\end{aligned}
$$

Hence $h_{2} o h_{1}$ is an isomorphism between $G_{1}^{*}$ and $G_{3}^{*}$.

Proposition 6 If $G_{1}^{*}, G_{2}^{*}, G_{3}^{*}$ are BVHFGs, then the weak isomorphism between these graphs is an partial order relation.

Proof Reflexivity is trivial. For anti-symmetry we assume a function $h: V_{1} \rightarrow V_{2}$ is a weak isomorphism on $G_{1}^{*}$ onto $G_{2}^{*}$ such that $h_{1}\left(m_{1}\right)=m_{2} \forall m_{1} \in V_{1}$ with conditions

$$
\begin{aligned}
& s\left(A_{1}\left(m_{1}\right)\right)=s\left(A_{2}\left(h_{1}\left(m_{1}\right)\right)\right), \\
& \quad s\left(B_{1}\left(m_{1} n_{1}\right)\right) \leq s\left(B_{2}\left(h_{1}\left(m_{1}\right) h_{1}\left(n_{1}\right)\right)\right) \\
& \forall m_{1} \in V_{1}, \forall m_{1} n_{1} \in \tilde{V}_{1}^{2} ;
\end{aligned}
$$

suppose $h_{2}: V_{2} \rightarrow V_{1}$ is a weak isomorphism between $G_{1}^{*}$ and $G_{2}^{*}$ such that $h_{2}\left(a_{2}\right)=a_{1} \forall a_{2} \in V_{2}$ with condition

$$
\begin{aligned}
& s\left(A_{2}\left(a_{2}\right)\right)=s\left(A_{1}\left(h_{2}\left(a_{2}\right)\right)\right), s\left(B_{2}\left(a_{2} b_{2}\right)\right) \\
& \quad \leq s\left(B_{2}\left(h_{2}\left(a_{2}\right) h_{2}\left(b_{2}\right)\right)\right) \forall a_{2} \in V_{2}, \\
& \forall a_{2} b_{2} \in \tilde{V}_{1}^{2} .
\end{aligned}
$$

From expressions (6) and (7), we conclude that both these inequalities hold if and only if the BVHFGs have the same number of edges and corresponding edges have the same weights, which shows that $G_{1}^{*}$ and $G_{2}^{*}$ are similar. Furthermore, we can show that the transitivity among the graphs $G_{1}^{*}, G_{2}^{*}$ and $G_{3}^{*}$ is the same as previous proposition.

Proposition 7 If $G^{*}=(A, B)$ is a $B V H F G$ and Aut $\left(G^{*}\right)$ is the set of all automorphisms of $G^{*}$. Then, $\left(\operatorname{Aut}\left(G^{*}\right), o\right)$ forms a group.

Proof For any $\phi, \psi \in A u t\left(G^{*}\right)$ and $x, y \in V$. Then, we have

$$
\begin{aligned}
& s(B((\phi o \psi)(x)(\phi o \psi)(y))) \\
& \quad=s(B(\phi(\psi(x))(\phi(\psi(y))) \\
& =s(B(\psi(x) \psi(y))) \\
& =s(B(x y)), \\
& s(A((\phi o \psi)(x)))=s(A(\phi(\psi(x)))) \\
& =s(A(\psi(x))) \\
& =s(A(x)),
\end{aligned}
$$

clearly $\phi о \psi \in \operatorname{Aut}\left(G^{*}\right)$. Also $\operatorname{Aut}\left(G^{*}\right)$ satisfies associative law under the mapping composition, let $I: G^{*} \rightarrow G^{*}$ be an identity mapping such that $\phi o I=I o \phi=\phi \forall \phi \in \operatorname{Aut}\left(G^{*}\right)$, also for each $\phi \in \operatorname{Aut}\left(G^{*}\right)$ there exists $\phi^{-1} \in G^{*}$ such t h a t $\quad s\left(A\left(\phi^{-1}(x)\right)\right)=s\left(A\left(\phi\left(\phi^{-1}(x)\right)\right)\right)=s(A((x)))$, $s\left(B\left(\phi^{-1}(x) \phi^{-1}(y)\right)\right)=s\left(B\left(\phi\left(\phi^{-1}(x)\right) \phi\left(\phi^{-1}(y)\right)\right)\right)=s(B(x y))$. Hence, $\left(\operatorname{Aut}\left(G^{*}\right), o\right)$ forms a group.

\section{Directed-BVHFGs and their application in decision making}

In this section, we present the definition of directed-BVHFGs and develop a method for finding in-degrees and outdegrees of vertices in directed-BVHFGs with the help of bipolar-valued hesitant fuzzy weighted averaging (BVHFWA) operator. Along with this, we have given a numerical example to find the domination degree of people in a meeting based on directed graph.

Definition 20 A directed-BVHFG $\vec{G}_{d}^{*}=(A, \vec{B})$ of the graph $G=(V, E)$ is given by the pair of membership functions $A: V \rightarrow P([0,1] \times[-1,0])$ and $\vec{B}: \tilde{V}^{2} \rightarrow P([0,1] \times[-1,0])$ with condition

$$
\begin{gathered}
s(\vec{B}(x y)) \leq s(A(x)) \wedge s(A(y)) \forall x y \in \tilde{V}^{2} \\
\text { and } s(\vec{B}(x y))=0 \forall x y \in\left(\tilde{V}^{2}-E\right) .
\end{gathered}
$$

Definition 21 Let $X$ be universe of discourse and $\left\{A_{i}(x) \mid x \in X, i=1,2 \cdots n\right\}$ be a collection of BVHFE's and $w=\left(w_{1}, w_{2} \cdots w_{n}\right)^{T}$ be the weight vector of $A_{i}(x)=\left(a_{i x}^{P}, a_{i x}^{N}\right)(i=1,2 \cdots n)$ with $w_{i} \in[0,1]$ and $\sum_{i=1}^{n} w_{i}=1$.

Then, bipolar-valued hesitant fuzzy weighted averaging (BVHFWA) operator is a mapping $B V H F W A: A^{n} \rightarrow A$, where

$$
\begin{aligned}
& \operatorname{BVHFWA}\left(A_{1}(x), A_{2}(x) \cdots A_{n}(x)\right)=\oplus_{i=1}^{n}\left(w_{i} \tilde{A}_{i}(x)\right) \\
& =\left\{\left(1-\prod_{i=1}^{n}\left(1-a_{i x}^{p}\right)^{w_{i}},-1+\prod_{i=1}^{n}\left(1+a_{i x}^{N}\right)^{w_{i}}\right) \mid\right. \\
& \left.a_{1 x} \in A_{1}(x), a_{2 x} \in A_{2}(x), \cdots a_{n x} \in A_{n}(x)\right\},
\end{aligned}
$$

here if $w=\left(\frac{1}{n}, \frac{1}{n}, \cdots \frac{1}{n}\right)^{T}$, then BVHFWA operator will become the bipolar-valued hesitant fuzzy averaging (BVHFA) operator. Equation (8) can be written as:

$$
\begin{gathered}
\operatorname{BVHFA}\left(A_{1}(x), A_{2}(x) \cdots, A_{n}(x)\right)=\oplus_{i=1}^{n}\left(\frac{1}{n} A_{i}(x)\right) \\
=\left\{\left(1-\prod_{i=1}^{n}\left(1-a_{i x}^{p}\right)^{\frac{1}{n}}, \quad-1+\prod_{i=1}^{n}\left(1+a_{i x}^{N}\right)^{\frac{1}{n}}\right) \mid\right. \\
\left.a_{1 x} \in A_{1}(x), a_{2 x} \in A_{2}(x), \cdots a_{n x} \in A_{n}(x)\right\} .
\end{gathered}
$$

Definition 22 Let $\mathbf{G}_{\mathbf{d}}^{*}=(A, \mathbf{B})$ be a directed-BVHFG over the graph $G=(V, E)$ and $v_{r}, r=1,2, \cdots n$ be adjacent BVHF-vertices of $v_{k} \in V$. With the help of Eq. (9), we 
define out-degree and in-degree of a vertex $v_{k}$ denoted by $O_{d}\left(v_{k}\right)$ and $I_{d}\left(v_{k}\right)$, respectively, and defined :

$O_{d}\left(v_{k}\right)=\left\{\left(1-\prod_{i=1}^{n}\left(1-b_{v_{k}, v_{i}}^{P}\right), \quad-1+\prod_{i=1}^{n}\left(1+b_{v_{k} v_{i}}^{N}\right)\right) \mid v_{k} v_{i} \in B\right\}$,

$I_{d}\left(v_{k}\right)=\left\{\left(1-\prod_{i=1}^{n}\left(1-b_{v_{i}, v_{k}}^{P}\right), \quad-1+\prod_{i=1}^{n}\left(1+b_{v_{i} v_{k}}^{N}\right)\right) \mid v_{i} v_{k} \in B\right\}$.

After finding the in-degree and out-degree of each vertex, we represent its score value by $s\left(O_{d}(v)\right)$ and $s\left(I_{d}(v)\right)$, respectively, and calculate it from definition 9.

To find the domination degree of each vertex $v_{k}$, we use $s\left(O_{d}\left(v_{k}\right)\right)-s\left(I_{d}\left(v_{k}\right)\right)$ where $s\left(O_{d}\left(v_{k}\right)\right)$ and $s\left(I_{d}\left(v_{k}\right)\right)$ represent the score value of out-degree and in-degree of vertex $v_{k}$, respectively, and denoted by $\omega\left(v_{k}\right)$.
Step 1: Let $\mathrm{A}=\left\{<p_{1},\{(0.40,-0.80),(0.50,-0.30),(0.90,-\right.$ $0.20)\}>,<p_{2},\{(0.30,-0.50),(0.60,-0.90)\}>,<p_{3},\{(0.10,-$ $0.60),(0.50,-0.20),(0.60,-0.40),(0.70,-0.40)\}>,<p_{4}$ $,\{(0.35,-0.60),(0.40,-0.80)\}>,<p_{5},\{(0.45,-0.30),(0.80,-$ $0.10),(0.75,-0.30)\}>,\left\langle p_{6},\{(0.60,-0.20),(0.90,-0.50)\}>\right.$, $\left.<p_{7},\{(0.20,-0.70),(0.55,-0.80),(0.80,-0.40)\}>\right\}$ be the BVHFS on the set $\mathrm{V}$ as in Table 2, which demonstrates the potential power of persons which are present in business meeting.

Step 2: Let $\mathbf{B}=\left\{<P_{1} P_{3},\{(.3,-.7),(.4,-.3),(.5,-.2)\}>\right.$, $<P_{1} P_{6},\{(.5,-.6),(.6,-.3)\}>,,<P_{1} P_{7},\{(.2,-.5),(.3,-.3),(.7,-$ $.4)\}>,\left\langle P_{2} P_{1},\{(.1,-.2),(.5,-.4),(.7,-.3)\}\right\rangle, \quad\left\langle P_{2} P_{3}\right.$ $,\{(.2,-.2),(.3,-.1),(.4,-.4),(.5,-.5)\}>,<P_{3} P_{7},\{(.45,-$ $.8)\}>,\left\langle P_{3} P_{4},\{(.1,-.5),(.5,-.9)\}>,\left\langle P_{4} P_{7},\{(.1,-.3),(.2,-\right.\right.$ $.5),(.3,-.2)\}>,\left\langle P_{5} P_{4},\{(.1,-.1),(.4,-.2),(.6,-.3)\}>\right.$, $<P_{5} P_{2},\{(.4,-.4),(.5,-.2)\}>,<P_{6} P_{3},\{(.2,-.1),(.3,-.2),(.5,-$ $.3),(.7,-.1)\}>,\left\langle P_{6} P_{4},\{(.1,-.1),(.2,-.5)\}>,<P_{6} P_{7},\{(.2,-\right.$ $.2),(.6,-.3),(.7,-.1)\}>,<P_{6} P_{2},\{(.2,-.3),(.5,-.2)\}>,<P_{7} P_{5}$

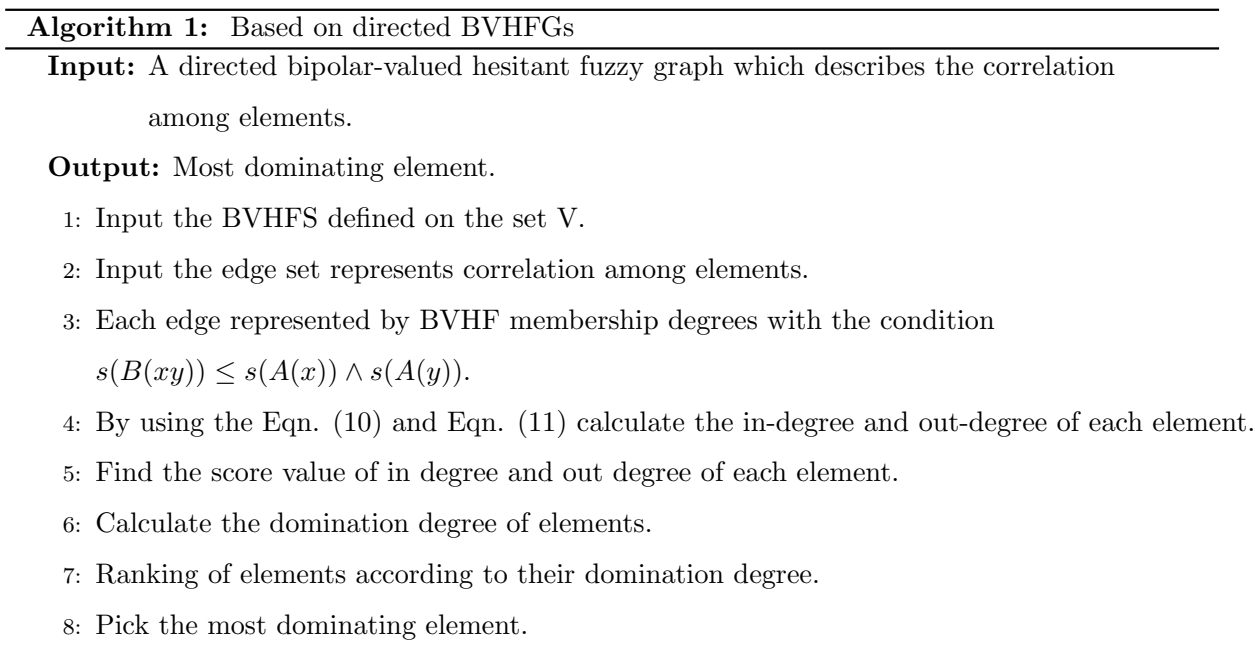

\subsection{Numerical example}

We cannot measure the impact power of person's properly, so we are always hesitant to evaluate the impact power of person's. Apart from this, if we do not know about a person properly, then we can also have a negative impact on him. In this part, we present a directed bipolar-valued hesitant fuzzy graph for such case.

Let us consider the directed-BVHFG of potential power of seven person's $P=\left\{p_{1}, p_{2} \cdots p_{7}\right\}$ in a business meeting. The potential power of person's determined in positive and negative membership degrees, positive hesitant membership degree denotes satisfaction degree of person corresponding to potential power, while negative hesitant membership degree denotes satisfaction degree of person to some implicit counter property of potential power.
$,\{(.3,-.2),(.4,-.3),(.5,-.1),(.8,-.1)\}>$,$\} be the set of bipolar-$ valued directed hesitant edges as in Table 3 , which demonstrates the impact power of one person onto another person in a meeting as in Fig. 3.

Step 3: With the help of Eqs. (10) and (11), we find the out-degree and in-degree of each person as in Table 4.

Step 4: Now we calculate the score value of in-degree and out-degree of each person by with the help of definition 8 and definition 9 as in Table 5.

Step 5: Finally, we calculate the domination degree using (Karaaslan 2019) of each person in the meeting as in Table 6.

Step 6: The ranking of each person present in the meeting based on their domination degree is as follows:

$P_{2}>P_{7}>P_{1}>P_{4}>P_{3}>P_{6}>P_{5}$, 
Table 2 BVHF membership of persons potential power

\begin{tabular}{lll}
\hline & $P_{1}$ & $P_{2}$ \\
\hline A & $\{(0.40,-0.80),(0.50,-0.30),(0.90,-0.20)\}$ & $\{(0.30,-0.50),(0.60,-0.90)\}$ \\
\hline & 0.516 & 0.575 \\
\hline & $P_{3}$ & $P_{4}$ \\
\hline$\{(0.10,-0.60),(0.50,-0.20),(0.60,-0.40),(0.70,-0.40)\}$ & $\{(0.35,-0.60),(0.40,-0.80)\}$ \\
& 0.437 & 0.537 \\
\hline$P_{5}$ & $P_{6}$ \\
\hline$\{(0.45,-0.30),(0.80,-0.10),(0.75,-0.30)\}$ & $\{(0.60,-0.20),(0.90,-0.50)\}$ \\
\hline 0.45. & 0.550 \\
\hline & $P_{7}$ \\
\hline
\end{tabular}

Table 3 Impact power of one person onto another person

\begin{tabular}{|c|c|c|}
\hline & $P_{1} P_{3}$ & $P_{1} P_{6}$ \\
\hline $\mathbf{B}$ & $\{(0.30,-0.70),(0.40,-0.30),(0.50,-0.20)\}$ & \}$\{(0.50,-0.60),(0.60,-0.30)\}$ \\
\hline \multirow[t]{4}{*}{ Score } & 0.40 & 0.50 \\
\hline & $P_{3} P_{7}$ & $P_{2} P_{1}$ \\
\hline & $\{(0.20,-0.50),(0.30,-0.30),(0.70,-0.40)\}$ & $\{(0.10,-0.20),(0.50,-0.40),(0.70,-0.30)\}$ \\
\hline & 0.40 & 0.367 \\
\hline$P_{2} P_{3}$ & $P_{5} P_{7}$ & $P_{3} P_{4}$ \\
\hline $\begin{array}{c}\{(0.20,-0.20),(0.30,-0.10) \\
\quad(0.40,-0.40),(0.50,-0.50)\}\end{array}$ & $\{(0.45,-0.80)\}$ & $\{(0.10,-0.50),(0.50,-0.90)\}$ \\
\hline \multirow[t]{7}{*}{0.365} & 0.625 & 0.5 \\
\hline & $P_{4} P_{7}$ & $P_{5} P_{4}$ \\
\hline & $\{(0.10,-0.30),(0.20,-0.50),(0.30,-0.20)\}$ & $\{(0.10,-0.10),(0.40,-0.20),(0.60,-0.30)\}$ \\
\hline & 0.267 & 0.283 \\
\hline & $P_{5} P_{2}$ & $P_{6} P_{3}$ \\
\hline & $\{(0.40,-0.40),(0.50,-0.20)\}$ & $\{(0.20,-0.10),(0.30,-0.20),(0.50,-0.30),(0.70,-0.10)\}$ \\
\hline & 0.375 & 0.30 \\
\hline$P_{6} P_{4}$ & $P_{6} P_{7}$ & $P_{6} P_{2}$ \\
\hline$\{(0.10,-0.10),(0.20,-0.50)\}$ & $\{(0.20,-0.20),(0.60,-0.30),(0.70,-0.10)\}$ & $\{(0.20,-0.30),(0.50,-0.20)\}$ \\
\hline \multirow[t]{3}{*}{0.225} & 0.35 & 0.30 \\
\hline & & $P_{7} P_{5}$ \\
\hline & & $\begin{array}{l}\{(0.30,-0.20),(0.40,-0.30),(0.50,-0.10),(0.80,-0.10)\} \\
0.337\end{array}$ \\
\hline
\end{tabular}

it is clear that the most dominating person in the meeting is $P_{2}$.

\section{Comparative analysis}

In this section, we evaluate the importance of HFG and BVHFG in decision making methods.
Following Karaaslan (2019), the hesitant fuzzy graph (HFG) $\tilde{G}=(\mathcal{A}, \mathcal{G})$ of the graph $G=(V, E)$ is characterize by membership functions $\mathcal{A}: V \rightarrow P([0,1])$ and $\mathcal{G}: \tilde{V}^{2} \rightarrow P([0,1])$ with the condition

$$
\begin{gathered}
\delta(\mathcal{G}(x y)) \leq \delta(\mathcal{A}(x)) \wedge \delta(\mathcal{A}(y)) \forall x y \in \tilde{V}^{2} \\
\text { and } \left.\delta(\mathcal{G}(x y))=0 \forall x y \in \tilde{(} V^{2}-E\right) .
\end{gathered}
$$




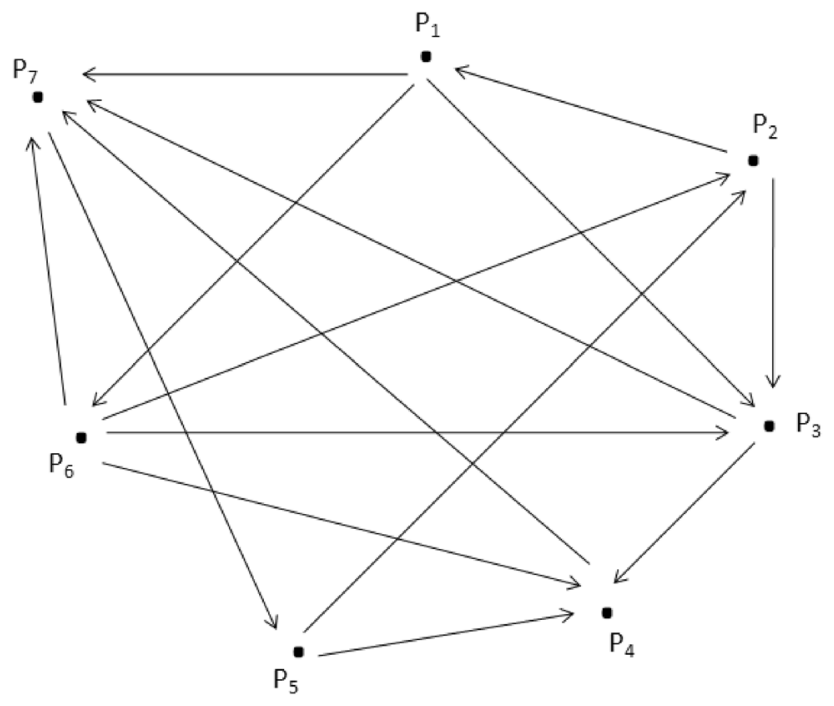

Fig. 3 Directed-BVHFG

where $\delta$ is the score valued function given by $\mathrm{Xia}$ and $\mathrm{Xu}$ (2011) such that $\delta(\mathcal{A}(x))=\frac{1}{l(\mathcal{A}(x))} \sum_{\gamma \in \mathcal{A}(x)} \gamma$, here $l(\mathcal{A}(x))$ represent the number of elements in $\mathcal{A}(x)$.

In HFG, all the informations is expressed with only positive membership degrees, which represents the satisfaction degree of an element corresponding to the set, and it ignores the degree of satisfaction of the element for some implicit counter property of the set. However, BVHFG simultaneously considers the positive and negative membership satisfaction degree, We will use the following tables for comparative study between BVHFG and HFG. In HFG, hesitant fuzzy table is composed only of the persons satisfaction degree corresponding to the set. Thus, hesitant fuzzy table of potential power of persons will only include the positive satisfaction degrees as in Table 7:
Based on the potential power of individuals, we establish the hesitant degree of impact power of one person to another person; here, the directed edge $P_{i} P_{j}$ represents impact power of $P_{i}$ on $P_{j}$ as in Table 8

Following Karaaslan (2019), we calculate the out-degree and in-degree of each person, by using the equations

$$
\begin{aligned}
O_{d}\left(v_{k}\right) & =\left\{\left(1-\prod_{i=1}^{n}\left(1-a_{v_{k}, v_{i}}\right)\right) \mid v_{k} v_{i} \in B\right\}, \\
I_{d}\left(v_{k}\right) & =\left\{\left(1-\prod_{i=1}^{n}\left(1-a_{v_{i}, v_{k}}\right)\right) \mid v_{i} v_{k} \in B\right\},
\end{aligned}
$$

which is given in Table 9.

Score value of in-degree and out-degree of each person is given in Table 10

Now we evaluate the domination degree of each person as in Table 11.

Through HFG domination degree of each person in this meeting is ranked as follows:

$P_{7}>P_{2}>P_{4}>P_{1}>P_{6}>P_{5}>P_{3}$,

clearly the person $P_{7}$ is the most dominating person in the meeting.

When the results of HFG and BVHFG are examined, we realize that the domination degree and ranking of dominating persons change significantly in two cases. In the BVHFG, $P_{2}$ is the most dominating person in the meeting, while in HFG the person $P_{7}$ is the most dominating person in the meeting. Furthermore, when we examine the ranking of persons in two cases, significant difference between two results is observed.

The main reason for this difference is the capability of BVHFG, and it is simultaneously considering the positive and negative membership degree with no restriction while the HFG considering only positive membership values.

Table 4 Out-degree and in-degree of each person

$$
\begin{aligned}
& O_{d}\left(P_{1}\right)=\{(0.79,-0.832),(0.8,-0.72),(0.832,-0.79)\} \\
& O_{d}\left(P_{2}\right)=\{(0.865,-0.664),(0.832,-0.784)\} \\
& O_{d}\left(P_{3}\right)=\{(0.45,-0.8),(0.55,-0.95)\} \\
& O_{d}\left(P_{4}\right)=\{(0.496,-0.72)\} \\
& O_{d}\left(P_{5}\right)=\{(0.784,-0.496),(0.7,-0.52)\} \\
& O_{d}\left(P_{6}\right)=\{(0.916,-0.546),(0.28,-0.55),(0.904,-0.496),(0.6,-0.44)\} \\
& O_{d}\left(P_{7}\right)=\{(0.958,-0.546)\} \\
& I_{d}\left(P_{1}\right)=\{(0.865,-0.664)\} \\
& I_{d}\left(P_{2}\right)=\{(0.7,-0.52),(0.6,-0.44)\} \\
& I_{d}\left(P_{3}\right)=\{(0.79,-0.832),(0.832,-0.784),(0.916,-0.546)\} \\
& I_{d}\left(P_{4}\right)=\{(0.55,-0.95),(0.784,-0.496),(0.28,-0.55)\} \\
& I_{d}\left(P_{5}\right)=\{(0.958,-0.664)\} \\
& I_{d}\left(P_{6}\right)=\{(0.8,-0.72)\} \\
& I_{d}\left(P_{7}\right)=\{(0.832,-0.79),(0.45,-0.8),(0.496,-0.72),(0.904,-0.496)\}
\end{aligned}
$$


Table 5 Score value of in-degree and out-degree

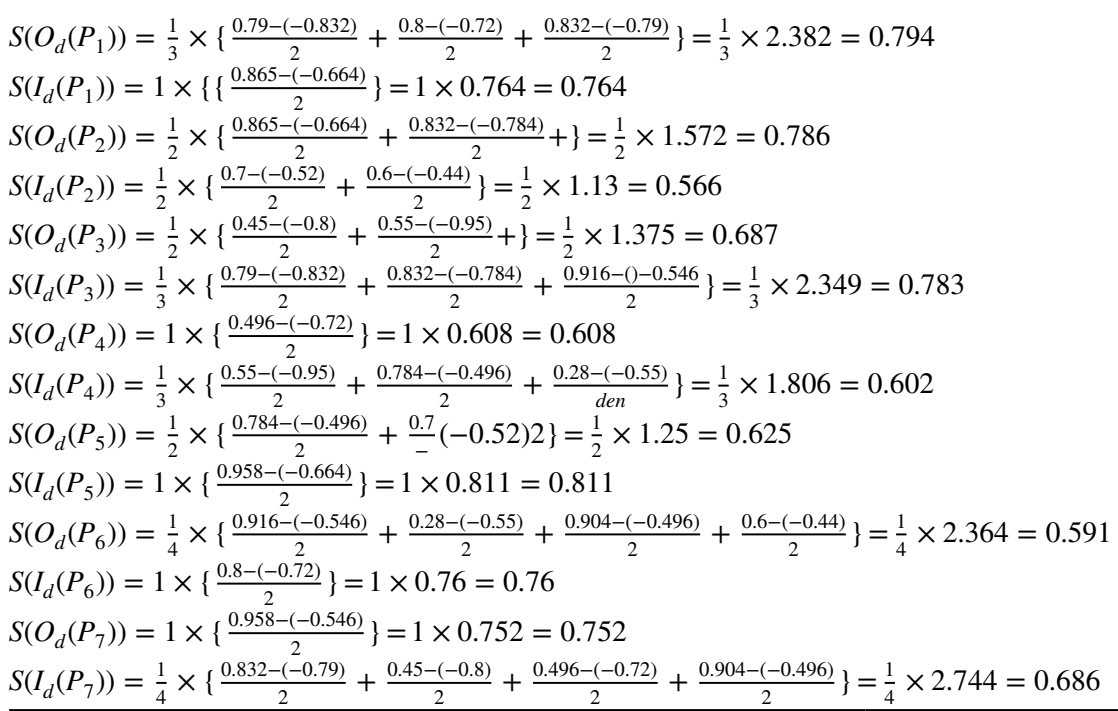

Table 6 Dominant degree of each person

\begin{tabular}{llc}
\hline Persons & $I_{d}-O_{o}$ & Dominant degree \\
\hline$\omega\left(P_{1}\right)$ & $0.794-0.764$ & 0.030 \\
$\omega\left(P_{2}\right)$ & $0.786-0.566$ & 0.220 \\
$\omega\left(P_{3}\right)$ & $0.687-0.783$ & -0.096 \\
$\omega\left(P_{4}\right)$ & $0.608-0.602$ & 0.006 \\
$\omega\left(P_{5}\right)$ & $0.625-0.811$ & -0.186 \\
$\omega\left(P_{6}\right)$ & $0.591-0.760$ & -0.169 \\
$\omega\left(P_{7}\right)$ & $0.752-0.686$ & 0.066 \\
\hline
\end{tabular}

\section{Conclusion}

In this paper, we have introduced the concept of BVHFG, some operations and propositions related to BVHFGs, such as Cartesian product, strong product, union, homomorphism, and isomorphism between BVHFGs. We have also investigated the concept of directed-BVHFG, which is a more helpful tool to express the various decision making problems. Furthermore, we have proposed a problem to find most dominating person in a meeting through directed-BVHFG. In the process of solving the problem, we construct a way to find the in-degree and out-degree of vertices, and then, we find out the score value of in-degree and out-degree of vertices, and then we calculate domination degree of persons.

In the final section, we point to comparative analyses between BVHFG and HFG. When we compare the outcome of hesitant fuzzy graph and bipolar-valued hesitant fuzzy graph, we notice the valuable difference between these two results. From the numerical illustrations, we conclude that bipolar-valued hesitant fuzzy graph provides more accurate results.
Table 7 Hesitant fuzzy membership of each person

\begin{tabular}{lll}
\hline Person & Hesitant fuzzy membership & Score value \\
\hline$P_{1}$ & $\{0.4,0.5,0.9\}$ & 0.6 \\
$P_{2}$ & $\{0.3,0.6\}$ & 0.45 \\
$P_{3}$ & $\{0.1,0.5,0.6,0.7\}$ & 0.457 \\
$P_{4}$ & $\{0.35,0.40\}$ & 0.357 \\
$P_{5}$ & $\{0.45,0.8,0.75\}$ & 0.667 \\
$P_{6}$ & $\{0.6,0.9\}$ & 0.75 \\
$P_{7}$ & $\{0.2,0.55,0.8\}$ & 0.517 \\
\hline
\end{tabular}

Table 8 Influence power of one person onto another person

\begin{tabular}{lll}
\hline Edge & Hesitant fuzzy membership & Score value \\
\hline$P_{1} P_{3}$ & $\{0.3,0.4,0.5\}$ & 0.4 \\
$P_{1} P_{6}$ & $\{0.5,0.6\}$ & 0.55 \\
$P_{1} P_{7}$ & $\{0.2,0.3,0.7\}$ & 0.4 \\
$P_{2} P_{1}$ & $\{0.1,0.5,0.7\}$ & 0.433 \\
$P_{2} P_{3}$ & $\{0.2,0.3,0.4,0.5\}$ & 0.350 \\
$P_{3} P_{4}$ & $\{0.45\}$ & 0.450 \\
$P_{3} P_{7}$ & $\{0.1,0.5\}$ & 0.300 \\
$P_{4} P_{7}$ & $\{0.1,0.2,0.3\}$ & 0.200 \\
$P_{5} P_{4}$ & $\{0.1,0.4,0.6\}$ & 0.367 \\
$P_{5} P_{2}$ & $\{0.4,0.5\}$ & 0.450 \\
$P_{6} P_{3}$ & $\{0.2,0.3,0.5,0.7\}$ & 0.425 \\
$P_{6} P_{4}$ & $\{0.1,0.2\}$ & 0.15 \\
$P_{6} P_{2}$ & $\{0.2,0.6,0.7\}$ & 0.500 \\
$P_{6} P_{7}$ & $\{0.2,0.5\}$ & 0.350 \\
$P_{7} P_{5}$ & $\{0.3,0.4,0.5,0.8\}$ & 0.500 \\
\hline
\end{tabular}


Table 9 Out-degree and in-degree of each person

\begin{tabular}{ll}
\hline$O_{d}\left(P_{1}\right)=\{0.79,0.8,0.832\}$ & $I_{d}\left(P_{1}\right)=\{0.865\}$ \\
$O_{d}\left(P_{2}\right)=\{0.865,0.832\}$ & $I_{d}\left(P_{2}\right)=\{0.7,0.6\}$ \\
$O_{d}\left(P_{3}\right)=\{0.45,0.55\}$ & $I_{d}\left(P_{3}\right)=\{0.79,0.832,0.916\}$ \\
$O_{d}\left(P_{4}\right)=\{0.496\}$ & $I_{d}\left(P_{4}\right)=\{0.55,0.784,0.28\}$ \\
$O_{d}\left(P_{5}\right)=\{0.784,0.7\}$ & $I_{d}\left(P_{5}\right)=\{0.958\}$ \\
$O_{d}\left(P_{6}\right)=\{0.916,0.28,0.904,0.6\}$ & $I_{d}\left(P_{6}\right)=\{0.8\}$ \\
$O_{d}\left(P_{7}\right)=\{0.958\}$ & $I_{d}\left(P_{7}\right)=\{0.832,0.45,0.496,0.904\}$ \\
\hline
\end{tabular}

Table 10 Score value of out-degree and in-degree

\begin{tabular}{ll}
\hline$\delta\left(O_{d}\left(P_{1}\right)\right)=0.807$ & $\delta\left(I_{d}\left(P_{1}\right)\right)=0.865$ \\
$\delta\left(O_{d}\left(P_{2}\right)\right)=0.849$ & $\delta\left(I_{d}\left(P_{2}\right)\right)=0.650$ \\
$\delta\left(O_{d}\left(P_{3}\right)\right)=0.500$ & $\delta\left(I_{d}\left(P_{3}\right)\right)=0.846$ \\
$\delta\left(O_{d}\left(P_{4}\right)\right)=0.496$ & $\delta\left(I_{d}\left(P_{4}\right)\right)=0.538$ \\
$\delta\left(O_{d}\left(P_{5}\right)\right)=0.742$ & $\delta\left(I_{d}\left(P_{5}\right)\right)=0.958$ \\
$\delta\left(O_{d}\left(P_{6}\right)\right)=0.675$ & $\delta\left(I_{d}\left(P_{6}\right)\right)=0.800$ \\
$\delta\left(O_{d}\left(P_{7}\right)\right)=0.958$ & $\delta\left(I_{d}\left(P_{7}\right)\right)=0.671$ \\
\hline
\end{tabular}

Table 11 Dominant degree of each person

\begin{tabular}{lll}
\hline$\omega\left(P_{1}\right)$ & $0.807-0.865$ & -0.058 \\
\hline$\omega\left(P_{2}\right)$ & $0.849-0.650$ & 0.199 \\
$\omega\left(P_{3}\right)$ & $0.500-0.846$ & -0.034 \\
$\omega\left(P_{4}\right)$ & $0.807-0.865$ & -0.042 \\
$\omega\left(P_{5}\right)$ & $0.807-0.865$ & -0.216 \\
$\omega\left(P_{6}\right)$ & $0.807-0.865$ & -0.125 \\
$\omega\left(P_{7}\right)$ & $0.807-0.865$ & 0.287 \\
\hline
\end{tabular}

\section{References}

Akram M (2011) Bipolar fuzzy graphs. Inf Sci 181(24):5548-5564 Akram M (2013) Bipolar fuzzy graphs with applications. Knowl-Based Syst 39:1-8

Akram M, Dudek WA (2012) Regular bipolar fuzzy graphs. Neural Comput Appl 21(1):197-205

Akram M, Akmal R (2016) Application of bipolar fuzzy sets in graph structures. Appl Comput Intell Soft Comput

Akram M, Waseem N (2018) Novel applications of bipolar fuzzy graphs to decision making problems. J Appl Math Comput 56(1):73-91

Akram M, Sarwar M, Dudek WA (2021) Graphs for the analysis of bipolar fuzzy information, vol 401. Springer, Berlin

Akram M, Alshehri N, Davvaz B, Ashraf A (2016) Bipolar fuzzy digraphs in decision support systems. J Multip Val Log Soft Comput 27(5-6):553-572

Alghamdi M, Alshehri NO, Akram M (2018) Multi-criteria decisionmaking methods in bipolar fuzzy environment. Int J Fuzzy Syst 20(6):2057-2064

Bhutani KR (1989) On automorphisms of fuzzy graphs. Pattern Recogn Lett 9(3):159-162

Bollobás B (2013), Modern graph theory, Vol. 184, Springer Science $\&$ Business Media, Berlin

Deepak D, John SJ (2014) Homomorphisms of hesitant fuzzy subgroups. Int J Sci Eng Res 59:9-14

Karaaslan F (2019) Hesitant fuzzy graphs and their applications in decision making. J Intell Fuzzy Syst 36(3):2729-2741

Kauffman A (1973) Introduction a La Theorie Des Sous-emsembles Flous, Masson et Cie Editures
Klir GJ, Yuan B (1996) Fuzzy sets, fuzzy logic, and fuzzy systems: selected papers by Lotfi A Zadeh, vol 6. World Scientific, Singapore

Mahapatra R, Samanta S, Allahviranloo T, Pal M (2019) Radio fuzzy graphs and assignment of frequency in radio stations. Comput Appl Math 38(3):1-20. https://doi.org/10.1007/ s40314-019-0888-3

Mahapatra R, Samanta S, Pal M, Xin Q (2019) Rsm index: a new way of link prediction in social networks. J Intell Fuzzy Syst 37(2):2137-2151. https://doi.org/10.3233/JIFS-181452

Mahapatra R, Samanta S, Bhadoria RS, Pal M, Allahviranloo T, Pandey B (2020) A graph networks based quality control model for packaged food smart traceability \& communication. Eur J Mol Clin Med 7(6):2830-2848

Mahapatra R, Samanta S, Pal M, Xin Q (2020) Link prediction in social networks by neutrosophic graph. Int J Comput Intell Syst 13:1699-1713

Mahapatra R, Samanta S, Pal M (2020) Applications of edge colouring of fuzzy graphs. Informatica 31(2):313-330

Mahapatra R, Samanta S, Pal M (2021) Generalized neutrosophic planar graphs and its application. J Appl Math Comput 65:693-712

Mahapatra R, Samanta S, Pal M, Lee J-G, Khan SK, Naseem U, Bhadoria RS (2021) Colouring of covid-19 affected region based on fuzzy directed graphs. Comput Mater Continua 68(1):1219-1233

Mandal P, Ranadive AS (2019) Hesitant bipolar-valued fuzzy sets and bipolar-valued hesitant fuzzy sets and their applications in multiattribute group decision making. Granular Comput 4(3):559-583

Mordeson JN, Nair PS (2012) Fuzzy graphs and fuzzy hypergraphs. Physica Vol. 46

Poulik S, Ghorai G (2020) Note on "bipolar Fuzzy Graphs with Applications". Knowl-Based Syst 192:105315

Rashmanlou H, Samanta S, Pal M, Borzooei RA (2015) A study on bipolar fuzzy graphs. J Intell Fuzzy Syst 28(2):571-580

Rosenfeld A (1975), Fuzzy graphs. In: Fuzzy sets and their applications to cognitive and decision processes, Elsevier , pp 77-95

Samanta S, Pal M (2012a) Bipolar fuzzy hypergraphs. Int J Fuzzy Log Syst 2(1):17-28

Samanta S, Pal M (2012b) Irregular bipolar fuzzy graphs, arXiv preprint arXiv: 1209.1682

Samanta S, Pal M, Pal M (2014) Some more results on bipolar fuzzy sets and bipolar fuzzy intersection graphs. J Fuzzy Math 22(2):253-262

Torra V (2010) Hesitant fuzzy sets. Int J Intell Syst 25(6):529-539

Xia M, Xu Z (2011) Hesitant fuzzy information aggregation in decision making. Int J Approx Reason 52(3):395-407

Zhang WR (1994) Bipolar fuzzy sets and relations: a computational framework for cognitive modeling and multiagent decision analysis. In: NAFIPS/IFIS/NASA'94. Proceedings of the first international joint conference of the North American fuzzy information processing society biannual conference. The Industrial Fuzzy Control and Intellige, IEEE, 1994, pp 305-309

Publisher's Note Springer Nature remains neutral with regard to jurisdictional claims in published maps and institutional affiliations. 